\title{
The Effect of the Moufarrege Total Posterior Pedicle Reduction Mammaplasty on the Erogenous Sensation of the Nipple
}

\author{
Richard Moufarrege ${ }^{1,2^{*}}$, Mohammed El Mehdi El Yamani', Laura Barriault ${ }^{1}$, \\ Ahmed Amine Alaoui ${ }^{1}$ \\ ${ }^{1}$ Faculty of Medicine, Université de Montréal, Montreal, Canada \\ ${ }^{2}$ Department of Plastic Surgery, Université de Montréal, Montreal, Canada \\ Email: *plasticsurg_moufar@hotmail.com
}

How to cite this paper: Moufarrege, R., El Yamani, M.E.M., Barriault, L. and Alaoui, A.A. (2019) The Effect of the Moufarrege Total Posterior Pedicle Reduction Mammaplasty on the Erogenous Sensation of the Nipple. Surgical Science, 10, 127-140. https://doi.org/10.4236/ss.2019.104016

Received: February 25, 2019

Accepted: April 23, 2019

Published: April 26, 2019

Copyright $\odot 2019$ by author(s) and Scientific Research Publishing Inc. This work is licensed under the Creative Commons Attribution International License (CC BY 4.0).

http://creativecommons.org/licenses/by/4.0/

\begin{abstract}
Traditional reduction mammoplasties have the simple concern to guarantee the survival of the nipple areola complex after surgery. Little has been done to take care of essential functions in the nipple, especially the erogenous sensation. We have conducted a retrospective study on a cohort of 573 female patients operated using the Total Posterior Pedicle of Moufarrege between 1985 and 1995 to evaluate its effect on the erogenous sensation of the nipple. This study demonstrated the preservation of the erogenous sensation of the nipple in a high proportion of these patients. The physiology of this preservation is explained in regard of the technique details in Moufarrege mammoplasty compared to other techniques. The Moufarrege Total Posterior Pedicle would therefore be a highly reliable reduction technique to ensure the preservation of the erogenous sensation of the nipple.
\end{abstract}

\section{Keywords}

Erogenous Sensation of the Nipple, Moufarrege Mammaplasty, Total

Posterior Pedicle Mammaplasty, Reduction Mammaplasty, Erogenous

Sensation

\section{Introduction}

It is common knowledge in the medical field that a reduction mammoplasty deprives the nipple from its erogenous sensation [1] [2]. This is the case in all breast reduction techniques other than the Total Posterior Pedicle. Only the Total Posterior Reduction technique of Moufarrege can guaranty, in almost 95\% of cases, that the erogenous sensation of the nipple will be preserved. This study is 
supported by a retrospective study over 573 patients having undergone a Moufarrege Total Posterior Pedicle reduction.

\section{Anatomy and Physiology}

Two types of sensation are possible within the nipple-areola complex.

\subsection{The Tactile Sensation of the Nipple}

This sensation is equivalent to the sensation of the entire cutaneous surface of the body. The sensory information is transmitted to the brain by the subcutaneous sensitive nerves [3].

In this case, the sensation of the nipple depends on the nerves that cover the cutaneous surface of the breast. These cutaneous nerves originate from the superficial cervical plexus. This plexus is composed of the great auricular, occipital, cervical transverse and supra-clavicular nerves. The branches of this plexus disperse in a subcutaneous path that ensures tactile sensation on the entire surface of the skin of the breast (Figure 1), including the areolar region [4]-[10].

\subsection{The Nipple's Erogenous Sensation}

The nipple's erogenous sensation is completely independent from the tactile sensation. This sensation is ensured by the intercostal nerves, essentially dorsal nerves IV, V and VI [11].

These nerves, after exiting from the vertebrae at levels four, five and six of the corresponding intercostal spaces, will follow a semi-circular path around the thorax. At the level of the posterior or median axillary line, they each form a subcutaneous branch, which crosses the muscle plane and then follows the aponeurosis of the thoracic muscles on the lateral side of the thorax in an areolar space until its arrival in the region of the vertical axis of the breast (Figure 2). From this point, these nerves enter the gland in a posterior-anterior trajectory and finally end at the nipple-areola complex and its adjacent region (Figure 3). Only these nerves are responsible for the erogenous sensation of the nipple [4]-[10].

When these nerves are interrupted, partially or completely, the nipple's erogenous sensation is impaired according to the degree of nerve damage [7] and, contrary to the tactile sensation nerves, this impairment is irreversible.

\section{Physiologic and Pathologic Variations}

Different variations of erogenous sensation exist at different stages in the general female population, outside of any surgical procedure or trauma [12].

Except for the variations in the general population where there is an absence of erogenous sensation in this region, our experience helped us realise that certain women, with very pronounced mammary hypertrophy, have lost, since mammary gland growth spurt, their nipples' erogenous sensation [13].

In our opinion, two factors can cause this loss of erogenous sensation: 


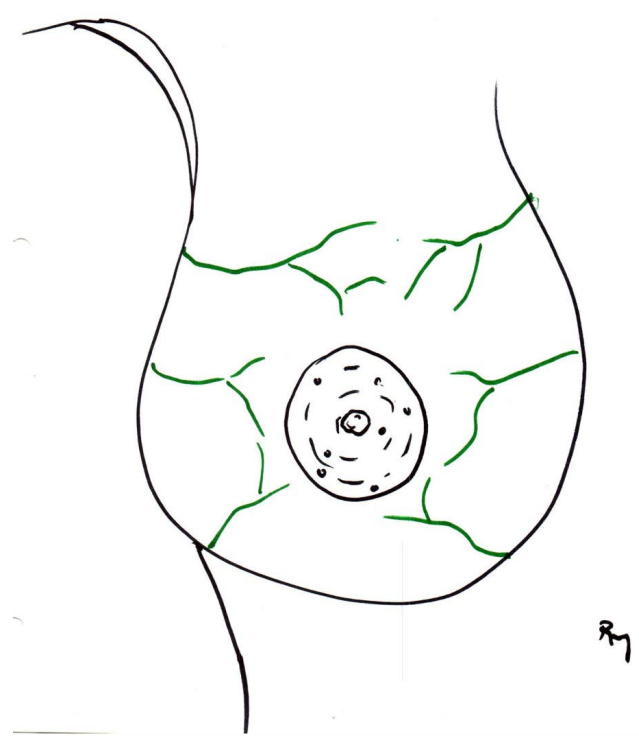

Figure 1. The superficial cervical plexus branches innervating the breast skin and providing tactile nipple sensation. Drawn by Dr. Richard Moufarrège.

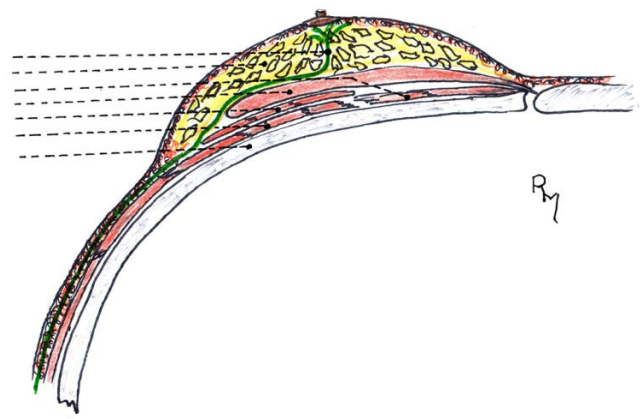

Figure 2. Path of the intercostal nerve from the medial axillary line to the center of the breast: I: Intercostal nerve; II: Breast gland; III: External oblique muscle; IV: Pectoralis major muscle; V: Pectoralis minor muscle; VI: Serratus anterior muscle; VII: Rib. Drawn by Dr. Richard Moufarrège.

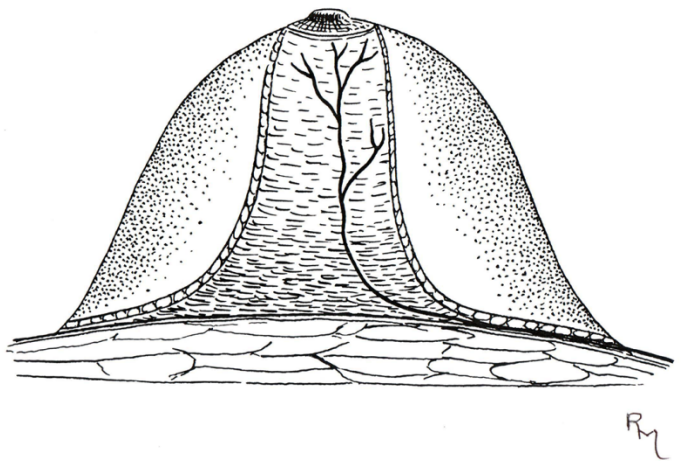

Figure 3. Intercostal nerve trajectory in the breast.Drawn by Dr. Richard Moufarrège.

\subsection{Psychological Factors}

Some women struggle to accept the mammary hypertrophy and therefore seem to be in denial of any aspect of their nipples' erogenous sensation [14] [15]. 


\subsection{Mechanical Factors}

Intercostal nerves 4, 5 and 6 may suffer from a neuropraxiasince they are stretched and put under stress from the abnormally high weight of the breasts. In certain cases, patients inform us that they have regained erogenous sensation in their nipples, which was previously lost long before the surgery [14] [15]!

Apart from the patients presenting a mammary hypertrophy, there remains, in the general population, a category of women that do not have an erogenous sensation of the nipple, for idiopathic reasons or reasons not yet determined [12]. According to several publications and studies, we can estimate that $21 \%$ of women do not have any erogenous sensation of the nipple, without ever having had any trauma or surgeries in this region [12].

\section{The Role of Mammaplasty in the Evolution of the Erogenous Sensation of the Nipple}

Knowledge of anatomy and of the nerves responsible for the erogenous sensation of the nipple enables us to understand the reasons why this sensation can be modified or preserved depending on the surgical technique used [16].

As previously described in this text, the three intercostal nerves (IV, V and VI) exit the vertebrae and, eventually, reach the posterior axillary line. At this level, these three nerves each divide into a subcutaneous branch, which crosses the muscle plane, following the thoracic muscles, and end in the central region of the breast (Figure 2 and Figure 3). It is from this region of the axillary line that the breast surgery can have an effect on these nerves [17] [18] [19].

The three intercostal nerves, IV, V and VI, responsible for the erogenous sensation of the nipple, follow a quarter-circle trajectory as they progress on the surface of the serratus anterior muscle and, minimally, on the small pectoral muscle (where the pectoralis minor musclelaterally exceeds the width of the pectoralis major muscle until the free border of the pectoralis major). Here, the three nerves pass in front of the pectoralis major and continue their path until they reach the central vertical axis of the breast before penetrating in front of the mammary gland to reach the nipple-areola complex (Figures 2-5) [17] [18] [19].

Any breast surgery that interrupts these nerves will have the consequence of modifying or deteriorating the erogenous sensation of the nipple. Of the 3 nerves mentioned, due to its terminal ending, the $5^{\text {th }}$ intercostal nerve has the highest likelihood of significantly decreasing erogenous sensation in this region when interrupted. Injury to one or two of these nerves do not condemn entirely the erogenous sensation but will impair it.

\section{The Future of the Erogenous Sensation of the Nipple in Breast Reduction}

The main element underlying the principle of the breast reduction technique is the pedicle of the nipple-areola complex. It is essentially this pedicle that will allow, or not, the three intercostal nerves (IV, V and VI) to innervate the nip- 
ple-areola complex [5] [6] [7] [8] [9].

Some pedicles have the potential to fulfill this requirement; others simply cannotpreserve the erogenous sensation of the nipple.

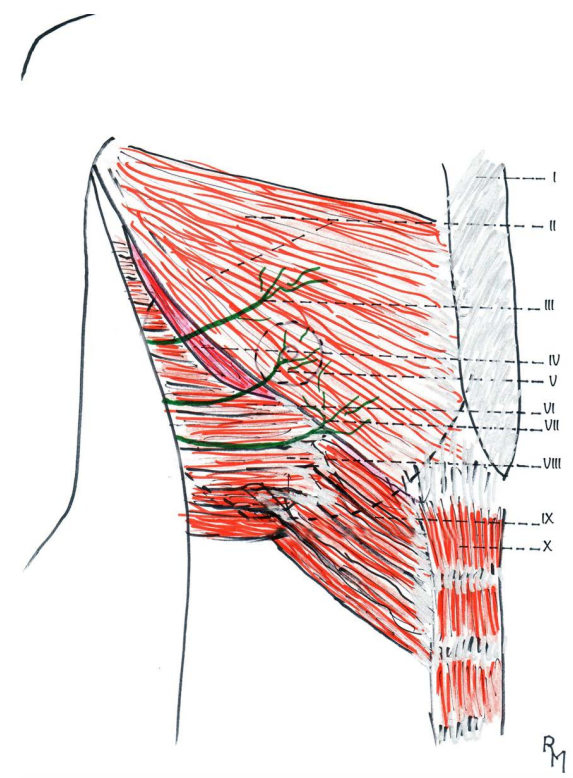

Figure 4. Trajectory of the three intercostal nerve IV, V and VI along the aponeurosis of the three muscles (Serratus anterior, Pectoralis minor and Pectoralis major): I: Sternum; II: Pectoralis major muscle; III: 4th intercostal nerve; IV: Pectoralis minor muscle; V: 5th intercostal nerve; VI: Serratus anterior muscle; VII: 6th intercostal nerve; VIII: Serratus anterior muscle; IX: External oblique muscle; X: Rectus abdominis muscle. Drawn by Dr. Richard Moufarrège.

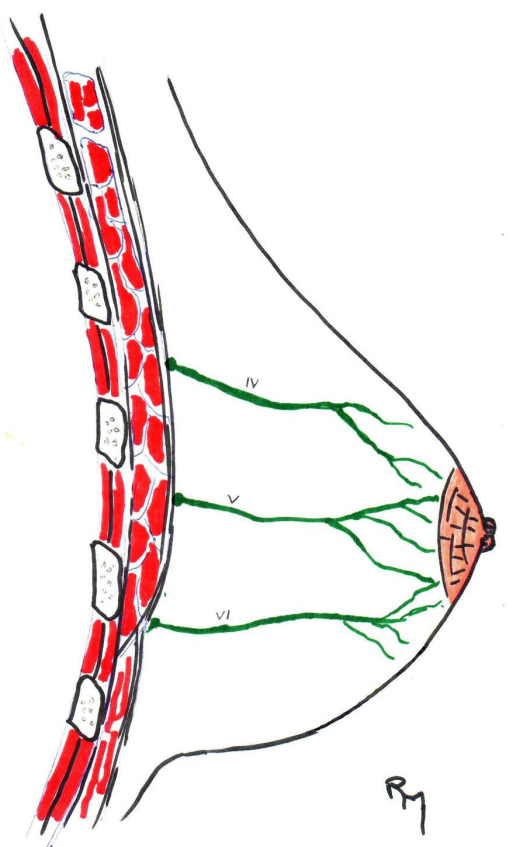

Figure 5. Sagittal section showing the trajectory of the three intercostal nerves IV, V and VI inside the breast gland. Drawn by Dr. Richard Moufarrège. 


\subsection{The Superior Pedicles}

All of the superior pedicles, regardless of their designation or inventor, cannot provide a path for the intercostal nerves IV, V and VI to reach the nipple-areola complex without being interrupted, because of their geography [12] [16]. This can easily be explained by the fact that these pedicles consist mostly of dermis, hypodermis and, sometimes, of a small piece of gland. Furthermore, these pedicles are located in a region and a position that in no way allows the aforementioned nerves to be included. It is thus out of the question to say that superior pedicles would have any capacity to ensure preservation of the nipple's erogenous sensation (Figure 6) [16]. With this type of pedicles, only tactile sensation could recover.

\subsection{The Inferior Pedicles}

The inferior pedicles (McKissock or Robbin types) could potentially serve as a bridge for one of the three intercostal nerves mentioned, most likely, the sixth intercostal nerve, which passes relatively low in the tissues of the lower quadrant of the breast [5]. Achieving this remains, however, only a possibility because of the variation between each surgeon and each operation. The inaccuracy associated with defining the limits of the pedicle could lead to the sixth intercostal nerve being included in some cases and excluded in others. The sixth intercostal nerve innervates the inferior aspect of the areola and its adjacent tissues. This nerve couldn't, in any case, ensure the true and complete erogenous sensation of the nipple. The Robbin (Figure 7) type inferior pedicle, which encompasses a slightly larger area than the McKissock (Figure 8) type, could be more likely to contain, in relatively rare cases, the fifth intercostal nerve, which would allow a better preservation of the erogenous sensation of the nipple. We insist that the chances of this preservation remain very slim.

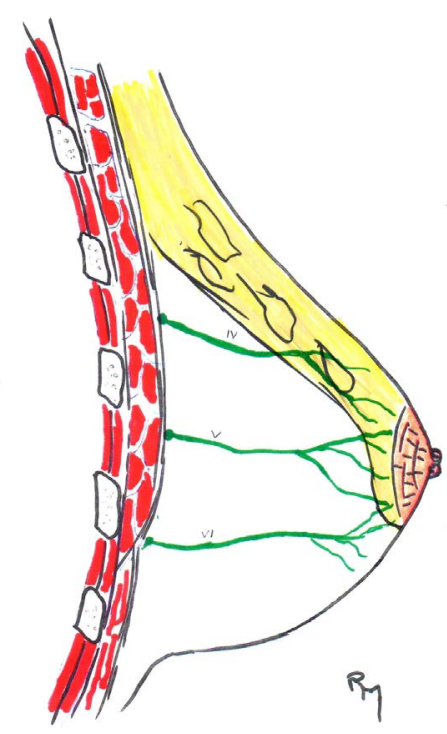

Figure 6. The superior pedicle cannot provide a secure pathway to any intercostal nerve, even not the 6thone. Drawn by Dr. Richard Moufarrège. 


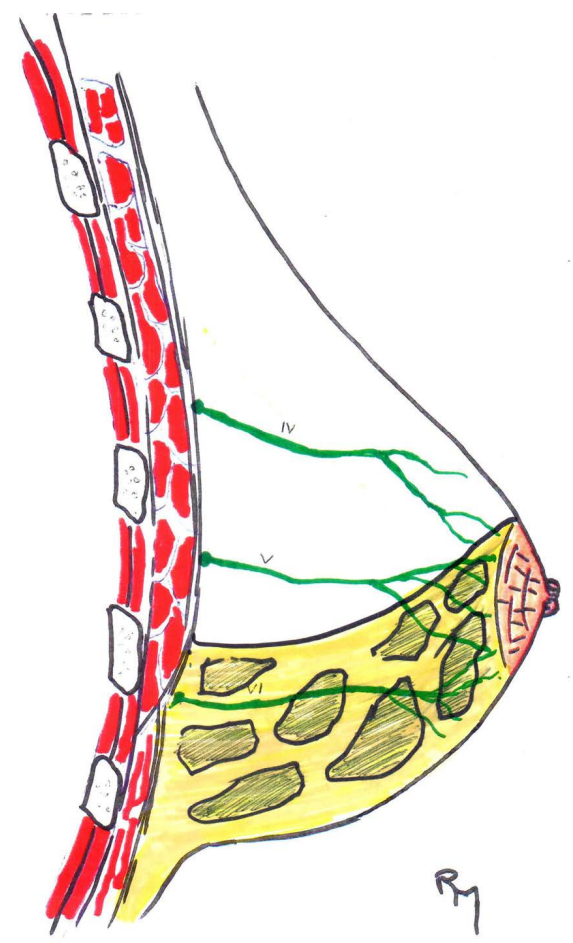

Figure 7. Sagittal section in Robbin's mammaplasty.Drawn by Dr. Richard Moufarrège.

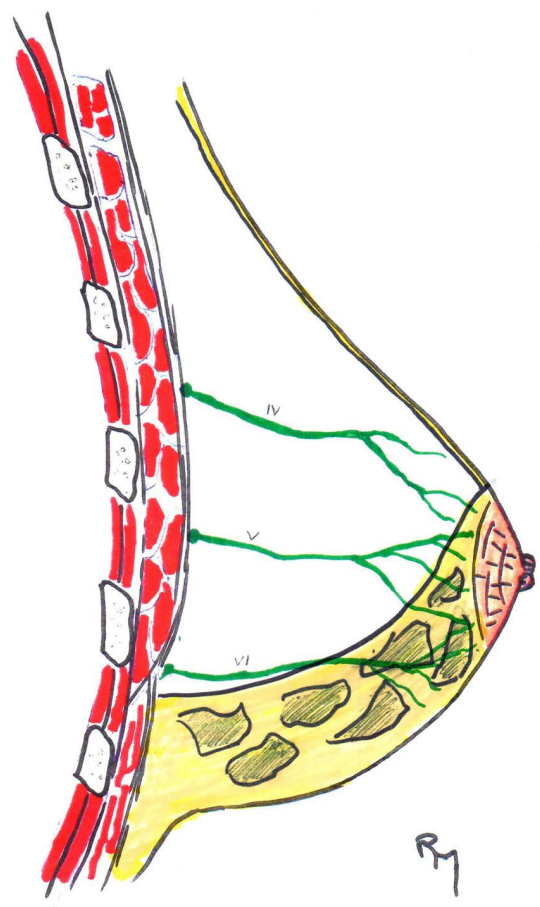

Figure 8. Sagittal section in McKissock's mammaplasty. Drawn by Dr. Richard Moufarrège.

\subsection{The Posterior Pedicles}

The posterior pedicles, under different designations, all derive from the Total Posterior Pedicle of Moufarrege, who was the first to describe the posterior 
pedicle, even if, in its beginning stages, this pedicle did not include the entire height of the breast [20].

These posterior pedicles, depending on the type, could possibly have the potential to spare one of the intercostal nerves (IV, V and VI) and preserve very partially the erogenous sensation of the nipple. However, depending on the type of pedicle and its dissection, there is a risk that the pedicle could be located beside the nerves and not include any of the three intercostal nerves mentioned above. There is also a chance that the pedicle could encompass the entire path of only one of these three nerves (Figure 9).

\subsection{The Total Posterior Pedicle of Moufarrege}

The Total Posterior Pedicle of Moufarrege can, if the technique is adequately executed, ensure an almost systematic preservation of the erogenous sensation of the nipple-areola complex [21]. We use the terms "almost systematic" because it is possible that the surgery performed is a slight variation of the technique described by Moufarrege [22]. This variation could be caused by the fact that the areolar tissue, which essentially covers the serratus anterior muscle, may not be preserved, contrarily to what is strongly recommended in the Moufarrege technique. Indeed, the intercostal nerves IV, V and VI pass through this areolar tissue to reach the central vertical axis of the breast before penetrating anteriorly in this tissue within the safe and large Total Posterior Pedicle (Figure 10 and Figure 11).

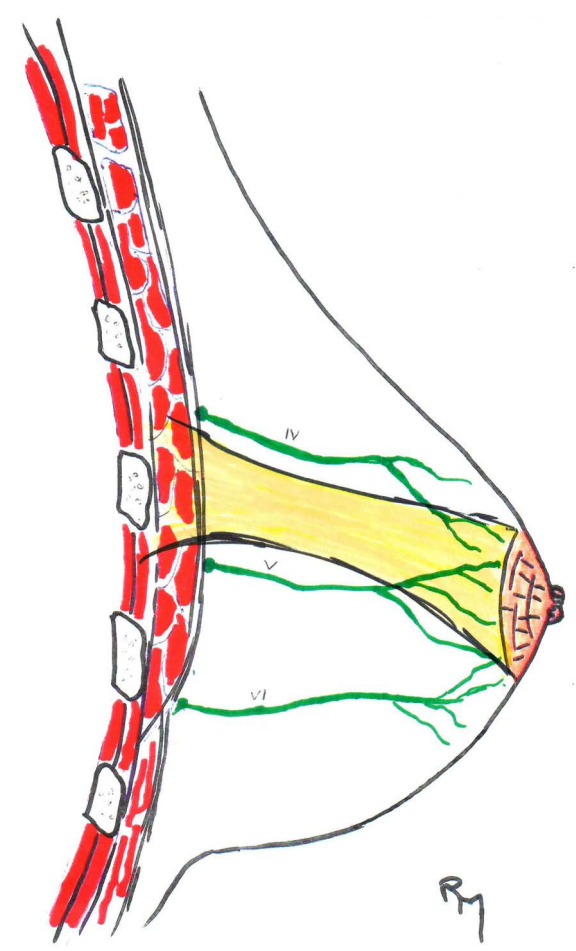

Figure 9. Sagittal section in Central Posterior pedicle technique.Drawn by Dr. Richard Moufarrège. 


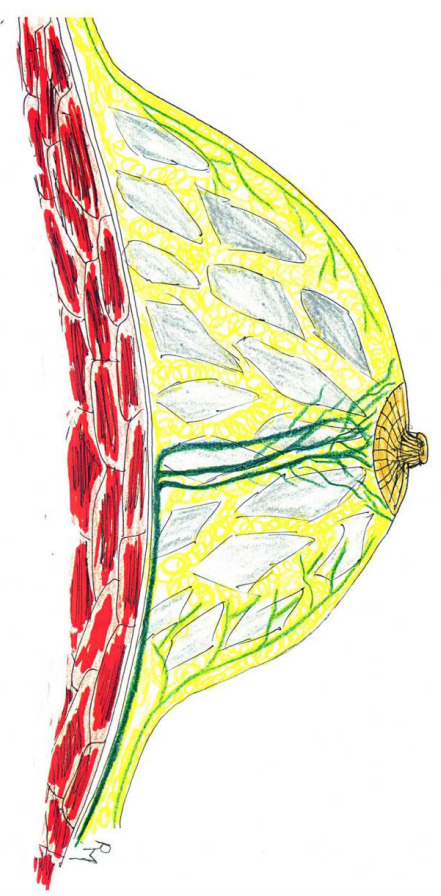

Figure 10. Transversal section in the Moufarrege Total Posterior pedicle.Drawn by Dr. Richard Moufarrège.

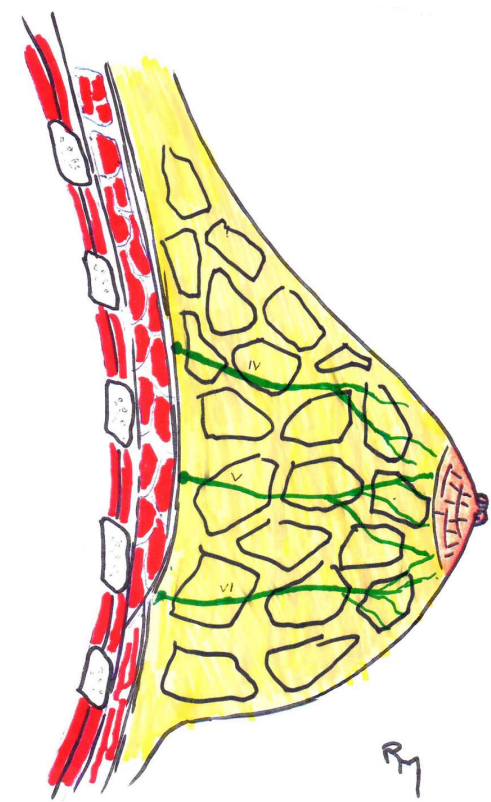

Figure 11. Sagittal section in the Moufarrege Total Posterior pedicle.Drawn by Dr. Richard Moufarrège.

\section{Retrospective Study of the Preservation of the Erogenous Sensation of the Nipple in Breast Reduction Using the Total Posterior Pedicle of Moufarrege}

We have conducted a retrospective study on a cohort of 573 female patients operated using the Total Posterior Pedicle of Moufarrege between 1985 and 1995. 
This study demonstrated the preservation of the erogenous sensation of the nipple in a relatively high proportion of patients. The Total Posterior Pedicle of Moufarrege would therefore essentially be the best breast reduction technique to ensure the preservation of the erogenous sensation of the nipple.

\subsection{Methods}

The population used for this retrospective study is a cohort of female patients having underwent a breast reduction using the technique of the Total Posterior Pedicle of Moufarrege between the years 1985 to 1995. This surgery was performed, in all cases, by the same surgeon. A total of 573 patients have accepted to participate in this study by completing a survey. All patients having answered the survey were included in the study without any exclusion. The primary objective of this study was to evaluate the degree of postoperative preservation of erogenous sensation of the nipple. In this survey, 5 values associated to a degree of erogenous sensation preservation were presented to the patients (preservation of $100 \%, 75 \%, 50 \%, 25 \%$ or $0 \%$ of the erogenous sensation of the nipple).

The overwhelming majority of patients included in this study are from French Canadian descent, much less from English Canadian descent.

This study has been approved by the Research Ethics Board of our institution and was conducted according to the principles of the Declaration of Helsinki.

\subsection{Results}

In the 573 patients who responded to the survey, 3 patients did not give an answer concerning erogenous sensation. Following their breast reduction, $70.4 \%$ (n $=401$ ) of patients reported that the erogenous sensation of their nipples was preserved at $75 \%-100 \%$. In $23.9 \%(n=136)$ of patients, the erogenous sensation was only preserved at $25 \%-50 \%$ compared to their preoperative sensation. Finally, $5.8 \%(\mathrm{n}=33)$ of the 570 patients report having lost all of their erogenous nipple sensation (Table 1).

\subsection{Discussion}

In light of these results, the Moufarrege Total Posterior pedicle reduction mammaplasty seems to be associated with highly favorable results in regard to the preservation of the erogenous sensation of the nipple.

It is extremely difficult, when reviewing the literature, to find studies on the proportion of women who have erogenous sensation in their nipples, regardless of any surgeries or trauma. However, regarding some publications on the erogenous sensation in the general population, we can infer that $79 \%$ of the general population would have erogenous sensation in their nipples [12].

In our study, the patients who considered that the erogenous sensation in their nipples was fully or almost fully preserved are $70.4 \%$ (Table 2). Patients having a partially preserved sensation account for $23.9 \%$ of all patients (Table 2). When added together, these values account for $94.3 \%$ of patients having 
Table 1. Degree of preservation of the erogenous sensation of the nipple in a cohort of 573 patients who underwent a Moufarrege Total Posterior pedicle breast reduction.

\begin{tabular}{ccc}
\hline $\begin{array}{c}\text { Percentage of preservation of erogenous } \\
\text { nipple sensation }\end{array}$ & Frequency & Percent \\
\hline $0 \%$ & 33 & 5.8 \\
$25 \%$ & 42 & 7.4 \\
$50 \%$ & 94 & 16.5 \\
$75 \%$ & 132 & 23.2 \\
$100 \%$ & 269 & 47.2 \\
Total & 570 & 100 \\
\hline
\end{tabular}

Table 2. Rationalization of the results of the preservation of the erogenous sensation of the nipple in the Moufarrege Total Posterior pedicle reduction.

\begin{tabular}{ccc}
\hline $\begin{array}{c}\text { Percentage of preservation of erogenous } \\
\text { nipple sensation }\end{array}$ & Frequency & Percent \\
\hline No sensation & 33 & 5.8 \\
Partial sensation & 136 & 23.9 \\
Full sensation & 401 & 70.4 \\
Total & 570 & 100 \\
\hline
\end{tabular}

Results are classed into three categories: full sensation (75\% - 100\%), partial sensation (25\% - 50\%) and no sensation (0\%).

conserved at least a partial erogenous sensation. Furthermore, almost $87 \%$ of our patients have maintained at least $50 \%$ of their erogenous sensation, which is higher than the value of $79 \%$ found in the literature [12].

It would have been interesting to obtain more information on the degree of erogenous sensation of the nipple present before breast reduction surgery. However, we have compared the postoperative erogenous sensation of the nipple of our patients with that of general population that never underwent any breast surgery. We also thoroughly tried to find an electrophysiological way to measure the conductivity of the erogenous sensation with possible electric devices (as an electromyography). Unfortunately, such tools do not exist and seem not realistically conceivable, so we could not but rely on the subjective evaluation of erogenous sensation of the patients. The high positive results in the erogenous sensation must be very reliable because, logically, no patient would attribute conservation of the erogenous sensation if there is big diminution or lack of the sensation.

Moreover, it can be noted that in some cases, patients recovered erogenous sensation of the nipple after a Moufarrege type breast reduction. Indeed, for some patients, an erogenous sensation which was absent prior to surgery appeared postoperatively. This can be explained by the suspected neuropraxiaimplicating the three intercostal nerves (IV, V and VI), due to the stretching effect caused by the weight of the mammary hypertrophy and of the resulting ptosis 
[14] [15]. Once this mechanical stressor is resolved, these nerves were able to function more efficiently and conduct the erogenous nervous influx to the brain.

\subsection{Conclusion}

The Moufarrege Total Posterior pedicle shows the highest preservation rate of the erogenous sensation amongst all other traditional techniques.

\section{Funding}

This research received no specific grant from any funding agency in the public, commercial, or not-for-profit sectors.

\section{Availability of Data and Materials}

Our research data will be available upon request.

\section{Ethics Approval and Consent to Participate}

The study has been approved by the Research Ethics Board of our institution and was conducted according to the principles of the Declaration of Helsinki.

\section{Conflicts of Interest}

Richard Moufarrege, Mohammed El Mehdi El Yamani, Laura Barriault and Ahmed Amine Alaoui have no conflict of interest to declare.

\section{Submission Declaration and Verification}

APRS submission guidelines have been reviewed.

This work has not been published previously.

All authors have made a substantial contribution to this study.

All authors have approved this version to be published.

\section{References}

[1] Courtiss, E.H. and Goldwyn, R.M. (1976) Breast Sensation before and after Plastic Surgery. Plastic and Reconstructive Surgery, 58, 1-13. https://doi.org/10.1097/00006534-197608000-00039

[2] Kuzbari, R. and Schlenz, I. (2007) Reduction Mammaplasty and Sensitivity of the Nipple-Areola Complex: Sensuality versus Sexuality? Annals of Plastic Surgery, 58, 3-11. https://doi.org/10.1097/01.sap.0000250648.70177.4a

[3] Tortora, G.J. and Derrickson, B. (2011) Principles of Anatomy and Physiology. Wiley, Hoboken.

[4] Schlenz, I., Kuzbari, R., Gruber, H. and Holle, J. (2000) The Sensitivity of the Nipple-Areola Complex: An Anatomic Study. Plastic and Reconstructive Surgery, 105, 905-909. https://doi.org/10.1097/00006534-200003000-00012

[5] Sarhadi, N.S., Shaw-Dunn, J., Lee, F.D. and Soutar, D.S. (1996) An Anatomical Study of the Nerve Supply of the Breast, Including the Nipple and Areola. British Journal of Plastic Surgery, 49, 156-164. https://doi.org/10.1016/S0007-1226(96)90218-0 
[6] Sarhadi, N.S., Shaw-Dunn, J. and Soutar, D.S. (1997) Nerve Supply of the Breast with Special Reference to the Nipple and Areola: Sir Astley Cooper Revisited. Clinical Anatomy, 10, 283-288. https://doi.org/10.1002/(SICI)1098-2353(1997)10:4<283::AID-CA12>3.0.CO;2-G

[7] Ferreira, M.C., Costa, M.P., Cunha, M.S., Sakae, E. and Fels, K.W. (2003) Sensibility of the Breast after Reduction Mammaplasty. Annals of Plastic Surgery, 51, 1-5. https://doi.org/10.1097/01.SAP.0000054190.76311.1A

[8] O'Connell, R.L. and Rusby, J.E. (2015) Anatomy Relevant to Conservative Mastectomy. Gland Surgery, 4, 476-483.

[9] Opatt, D. and Morrow, M. (2006) The Dual Role of Nipple Preservation. The Journal of Supportive Oncology, 4, 233-234.

[10] Del Vecchyo, C., Caloca, J., Caloca, J. and Gomez-Jauregui, J. (2004) Evaluation of Breast Sensibility Using Dermatomal Somatosensory Evoked Potentials. Plastic and Reconstructive Surgery, 113, 1975-1983. https://doi.org/10.1097/01.PRS.0000122210.12819.B8

[11] Ramachandran, K. (2008) Breast Augmentation. Indian Journal of Plastic Surgery, 41, S41-S47.

[12] Schlenz, I., Rigel, S., Schemper, M. and Kuzbari, R. (2005) Alteration of Nipple and Areola Sensitivity by Reduction Mammaplasty: A Prospective Comparison of Five Techniques. Plastic and Reconstructive Surgery, 115, 743-751. https://doi.org/10.1097/01.PRS.0000152435.03538.43

[13] Temple, C.L. and Hurst, L.N. (1999) Reduction Mammaplasty Improves Breast Sensibility. Plastic and Reconstructive Surgery, 104, 72-76. https://doi.org/10.1097/00006534-199907000-00010

[14] Godwin, Y., Valassiadou, K., Lewis, S. and Denley, H. (2004) Investigation into the Possible Cause of Subjective Decreased Sensory Perception in the Nipple-Areola Complex of Women with Macromastia. Plastic and Reconstructive Surgery, 113, 1598-1606. https://doi.org/10.1097/01.PRS.0000117190.00235.5C

[15] Slezak, S. and Dellon, A.L. (1993) Quantitation of Sensibility in Gigantomastia and Alteration Following Reduction Mammaplasty. Plastic and Reconstructive Surgery, 91, 1265-1269. https://doi.org/10.1097/00006534-199306000-00012

[16] Tairych, G., Worseg, A., Kuzbari, R., Deutinger, M. and Holle, J. (2000) A Comparison of Long-Term Outcome of 6 Techniques of Breast Reduction. Handchirurgie-Mikrochirurgie-Plastische Chirurgie, 32, 159-165. https://doi.org/10.1055/s-2000-10926

[17] Riccio, C.A., Zeiderman, M.R., Chowdhry, S., Brooks, R.M., Kelishadi, S.S., Tutela, J.P., et al. (2015) Plastic Surgery of the Breast: Keeping the Nipple Sensitive. Eplasty, $15, \mathrm{e} 28$.

[18] Blondeel, P.N., Demuynck, M., Mete, D., Monstrey, S.J., Van Landuyt, K., Matton, G., et al. (1999) Sensory Nerve Repair in Perforator Flaps for Autologous Breast Reconstruction: Sensational or Senseless? British Journal of Plastic Surgery, 52, 37-44. https://doi.org/10.1054/bjps.1998.3011

[19] Jaspars, J.J., Posma, A.N., van Immerseel, A.A. and Gittenberger-de Groot, A.C. (1997) The Cutaneous Innervation of the Female Breast and Nipple-Areola Complex: Implications for Surgery. British Journal of Plastic Surgery, 50, 249-259. https://doi.org/10.1016/S0007-1226(97)91155-3

[20] See, M.H. (2014) Central Pedicle Reduction Mammoplasty: A Reliable Technique. Gland Surgery, 3, 51-54.

[21] Moufarrege, R., Beauregard, G., Bosse, J.P., Muller, G., Papillon, J. and Perras, C. 
(1985) Reduction Mammoplasty by the Total Dermoglandular Pedicle. Aesthetic Plastic Surgery, 9, 227-232. https://doi.org/10.1007/BF01570855

[22] Moufarrege, R. (2003) Moufarrege Total Posterior Pedicle Breast Reduction. eMedicine. https://emedicine.medscape.com/article/1275321-overview 